\title{
Challenges of the Dynamic Detection of Functionally Similar Code Fragments
}

\author{
Florian Deissenboeck, Lars Heinemann, Benjamin Hummel \\ Technische Universität München, Germany \\ \{deissenb,heineman,hummelb\}@in.tum.de
}

\author{
Stefan Wagner \\ University of Stuttgart, Germany \\ stefan.wagner@informatik.uni-stuttgart.de
}

\begin{abstract}
Classic clone detection approaches are hardly capable of finding redundant code that has been developed independently, i.e., is not the result of copy\&paste. To automatically detect such functionally similar code of independent origin, we experimented with a dynamic detection approach that applies random testing to selected chunks of code similar to Jiang\&Su's approach. We found that such an approach faces several limitations in its application to diverse Java systems. This paper details on our insights regarding these challenges of dynamic detection of functionally similar code fragments. Our findings support a substantiated discussion on detection approaches and serve as a starting point for future research.

Index Terms-dynamic analysis; functional similarity
\end{abstract}

\section{INTRODUCTION}

Research in software maintenance has shown that many programs contain a significant amount of duplicated (cloned) code. Such cloned code is considered harmful for two reasons: (1) multiple, possibly unnecessary, duplicates of code increase maintenance costs [1], [2] and, (2) inconsistent changes to cloned code can create faults and, hence, lead to incorrect program behavior [3]. The negative impact of clones on software maintenance is not due to copy\&paste but caused by the semantic coupling of the clones. Hence, functionally similar code, independent of its origin, suffers from the same problems clones are known for. In fact, the re-creation of existing functionality can be seen as even more critical, since it is a missed reuse opportunity.

The manual identification of functionally similar code is infeasible in practice due to the size of today's software systems. Tools are required to automatically detect similar code. An earlier study [4] has shown that existing clone detection tools are limited to finding duplicated code, i.e., they are not capable of finding redundant code that has been developed independently. As a result, we do not know to what degree real-world software systems contain similar code beyond the code clones that stem from copy\&paste programming. Manual analysis of sample projects [4] as well as anecdotal evidence, however, indicate that programs indeed contain many similarities not caused by copy\&paste. Hence, we expected that tools for the automatic detection of similar code could prove as beneficial for quality assurance activities as the now widely-used clone detection tools.

While the equivalence of two programs is undecidable in general, a straightforward approach to detect similar code relies on executing candidate code fragments with random input data and comparing the output values. This approach was pursued by Jiang\&Su [5] as well as us. While they successfully applied their approach for code in the Linux kernel, we were unable to produce significant results using this seemingly simple approach for diverse Java systems. As this differs from Jiang\&Su's results, this paper details on our insights regarding challenges of dynamic detection of functionally similar code in Java programs.

Research Problem: Functional duplication in software systems causes a multitude of problems for software maintenance. Although clone detection is a viable approach to find copied code, existing tools are not capable of finding similar code that was created independently [4]. Jiang\&Su [5] developed a dynamic approach to identify functionally similar code in $\mathrm{C}$ systems and experienced a high detection rate for the Linux kernel. We implemented a similar approach for detecting functionally similar code fragments in Java systems. When analyzing five open-source Java systems, we got considerably lower detection rates. We found this to be caused by limitations of the approach when applied to Java systems. As a consequence, it is unclear if the dynamic approach can be applied in practice to detect functionally similar code fragments in object-oriented systems.

Contribution: While not a replication in the strict sense, this paper transfers Jiang\&Su's work to object-oriented software implemented in Java. We describe our implementation of the dynamic detection approach, report on the detection results, and provide a detailed comparison of our approach and results to Jiang\&Su's work. Moreover, we discuss the challenges of the dynamic detection approach for object-oriented systems and thereby provide a basis for a substantiated discussion as well as directions for further research.

\section{TERms \& DeFInITIONS}

Simion: We define a simion as a functionally similar code fragment regarding I/O behavior [4]. Two fragments are simions if they compute the same output for all input values.

Chunk: A chunk is a code fragment that is compared for functional similarity. It consists of a set of input parameters, a statement sequence, and a set of output parameters.

Clone: We define a clone as a syntactically similar code fragment typically resulting from copy\&paste and potential modification. Several specific clone types were introduced that impose constraints on the differences between the code 
fragments [1]. Type-1 clones are clones that may differ in layout and comments. Type-2 clones may additionally differ in identifier names and literal values. Type-3 clones allow a certain amount of statement changes, deletions or insertions. Type-4 clones as defined by [2] are comparable to simions. However, we do not use the term type-4 clones, since the term "clone" implies that one instance is derived from the other, which is not necessarily the case for simions according to our definition. In addition to the known clone types we define for this paper type-1.5 clones as type- 1 clones that may be subject to consistent variable renaming. The clone types form an inclusion hierarchy, i.e., all type- 2 clones are also type- 3 clones, but there are type- 3 clones that are not type- 2 (and analogously for the other types). While clones may be (and often are) simions, even textually identical fragments of code may emit different behavior because of the type binding implied by the surrounding context.

\section{DYNAMIC DETECTION APPROACH}

Our approach for dynamically detecting functionally similar code fragments follows in principle that of Jiang\&Su [5], i. e., it is based on the fundamental heuristic that two functionally similar code fragments will produce the same output for the same randomly generated input. We exclude clones from the simion detection, however, since these can be found with existing clone detection tools. The main difference to the approach of Jiang\&Su is that our approach targets objectoriented systems written in Java whereas they address C programs. The detection procedure can be divided in five principal phases that are executed in a pipeline fashion. We implemented a prototype of this pipeline based on our continuous quality assessment toolkit ConQAT 1

Figure 1 illustrates the detection pipeline with its five phases, each consisting of several processing steps. The input of the pipeline is the source code of the analyzed projects and their referenced libraries. The output is a set of equivalence classes of functionally similar code fragments. The pipeline phases are detailed in the following sections.

\section{A. Chunking}

Chunk Extraction: This step extracts a set of chunks from each source code file, which are used as candidates for simion detection. For Java systems, which consist of classes, it is a challenge to extract those chunks, since a code fragment arbitrarily cut out of the source files will almost certainly not represent a compilable unit on its own. Therefore, we developed several strategies for extracting chunks from Java classes. The first challenge is to determine what will be the input and output parameters of the chunk. In a Java class, several types of variables occur. During the chunk extraction we derive the input and output parameters from the declared and referenced variables in the statements of the chunk with the following heuristics:

- Referenced instance variables (non-static) become input as well as output parameters.

\footnotetext{
${ }^{1}$ http://www.conqat.org
}

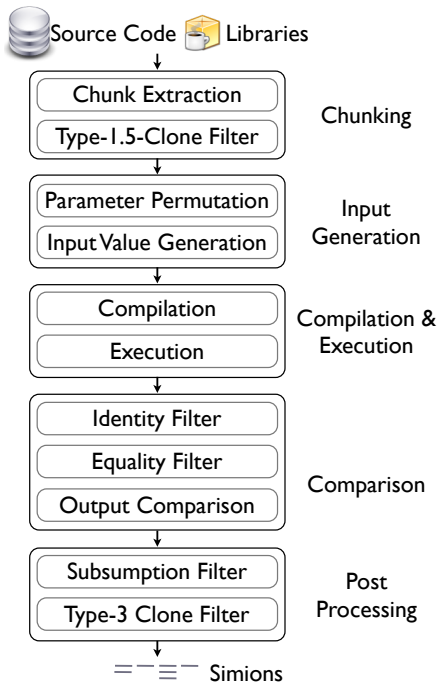

Fig. 1. Simion Detection Pipeline

- Referenced class variables (static) become neither input nor output variables, but are treated as a local variable as some of the called methods might rely on the value of these globals.

- Local variables with a scope that is nested in the statements of the chunk do not have to be considered as input or output variables. All other referenced local variables become output variables. Local variables that are referenced but not declared in the chunk statements also become input variables.

- Method parameters are treated in the same way as referenced but not declared local variables, i.e., they become input and output parameters. They are also output parameters, as the code after the chunk might reference these values.

Since we want to compare individual chunks for functional similarity, we have to be able to compile their statement sequences and execute them separately. We therefore process each statement sequence and apply several transformations to obtain a static function with the input and output parameters of the chunk. In certain cases, two chunks are generated for the same statement sequence. This is used to provide different input signatures for the same piece of code. For example, code referencing the attributes of the class could either use an input object of the class' type or inputs for each of the attributes, leading to different chunk signatures. There are cases when we cannot create a compilable chunk at all. Examples include statement sequences with branch statements (e.g., continue) where the target of the branch statement is not in the sequence or calls to constructors of non-static inner classes.

Non-static methods that could be static since they do not reference any non-static methods or fields are converted to static methods by adding the static keyword to the method declaration. Thereby potentially more chunks do not need an 
additional input parameter with the type of the surrounding class and thus represent a more generally reusable fragment.

In case that a statement sequence contains a return statement, the resulting chunk gets a single output parameter with the type of the return value of the associated method. The code with the return statement is changed to a local variable declaration with the corresponding initialization followed by a jump to an exit label.

The detection performance heavily depends on the number of chunks compared for similarity. Since Jiang\&Su experienced performance problems due to high numbers of chunks, we developed three different chunk extraction strategies.

The sliding window strategy extracts chunks by identifying all possible statement subsequences that represent valid AST fragments with a certain minimal length. Thus, it can cover cases where an arbitrary statement sequence is functionally similar to another one. However, the number of chunks is quadratic in the number of statements of a method.

The intent-based strategy utilizes the programmer's intent by interpreting blank lines or comments in statement sequences as a logical separator between functional units. It extracts one chunk that includes all statements in a method and all chunks that can be formed from the statement sequences separated by blank or comment lines.

The method-based strategy utilizes structuring of the code given by the methods and considers the statement sequences of all methods in the code. This strategy has a slight variation for determining the output parameters. If the method has a non-void return value the chunk gets one output parameter with the return type of the method. Otherwise the chunk gets one output parameter with the type of the surrounding class.

Type-1.5-Clone Filter: Although code clones are often simions w.r.t. to our definition, they are not useful as they could be more easily detected with a clone detection tool. The type-1.5-clone filter discards chunks that are type-1.5 clones. This removes the clones from the results and improves detection performance as these chunks are not further processed.

\section{B. Input Generation}

Parameter Permutation: To cover cases where two code fragments compute the same function but have different parameter ordering, we additionally apply a parameter permutation. For each chunk we generate additional chunks where all input parameters of the same type are permutated. To cope with combinatorial explosion, we constrain the number of additionally generated permutations to 25 .

Input Value Generation: As Jiang\&Su, we employ a random testing approach for generating input values. We generate code for constructing random values for the input parameters of the chunks. Since the chunks must be executed on the same inputs for comparison, the input generation uses a predefined set of values for primitive parameters (all Java primitives, their wrapper types, and String). Input values for parameters with composite types are generated with a recursive algorithm. All constructors (if any) of the type are used for creating instances. The algorithm is applied recursively to the parameters of the constructors, i.e., again, random values are generated and passed as arguments to the constructor. In case of 1-dimensional arrays and Java 5 collections where the component type is known, we generate collections of several randomly chosen but fixed sizes and apply the algorithm recursively for the elements of the collection. For chunks with many parameters or constructors with many arguments, a large number of inputs would be generated by this approach. Therefore, we constrain the number of input values generated for one chunk to 100 .

\section{Compilation \& Execution}

Compilation: The compilation step wraps the chunk code with the input generation code and code for handling errors during execution and for storing the values of the output parameters after execution. If an exception occurred during the execution, a special output value error is used. Moreover, we generate code for handling non-terminating chunks (e.g., due to infinite loops), which stops execution after a timeout of 1 second. The code is compiled in a static method within a copy of the original class code and with all compiled project code and libraries on the classpath. This means that the statements in the chunk have access to all static methods and fields from the original context as well as their dependencies.

Execution: After compilation, the chunks are executed in groups of at most 20 in a separate Java Virtual Machine with a security manager configured. This ensures that the chunk execution does not have unwanted side effects $(e . g$., deletion of files). The result of the execution step is a list of execution data objects, which hold data about the chunk and a list of input and output values of the chunk execution.

\section{Comparison}

Preliminary experiments with the simion detection pipeline revealed that the majority of identified simions is not relevant. A large fraction are false positives, i.e., our analysis identified them as functionally similar although they are not. This is caused by the random nature of the generated input data. Often two (or more) chunks are executed with input data that triggers only very specific execution paths. With respect to these paths the chunks are similar although they are not for reasonable input data. An example are two string processing methods where one trims the string (deletes leading and trailing whitespace) and one replaces characters with a unicode code point above 127 with the unicode escape sequence used in Java. If both chunks are executed for a string without leading or trailing whitespace and without characters outside the ASCII range, they both simply return the input string. As a result, they are identified as a simion. We address both problems, clones and false positives, with additional filter steps in our detection pipeline.

Identity filter: The identity filter discards all chunks that implement the identity function, i.e., for each input data set they return the same output data set. This heuristic excludes chunks for which the randomly generated input data is not capable of triggering interesting execution paths. 
Equality filter: The equality filter discards all chunks that generate the same output data for all input data sets. The rationale behind this is that the chunk execution is apparently independent of the input data. Again, this is caused by the inherently limited quality of the randomly generated input data. An example is a chunk that has two input parameters: a string and an integer value $i$. If $i$ is less than the length of the string (in characters), the chunk returns a new string with the first $i$ characters of the input string. If $i$ is greater than the length of the string, it returns the empty string. As this dependency between the two parameters is unknown to the input data generator it could possibly generate only data sets where $i$ is greater than the length of the chosen string. All executions of the chunk return the empty string.

Output Comparison: The output comparison step uses the execution data objects to compare the chunks for functional similarity. Chunks that do not provide valid output data for at least 3 inputs (i.e., either throw an exception or have a time-out), are discarded at this step. To make the comparison performant we use a hash-based approach. For each chunk and each of its output variables, it computes an MD5 digest for the comparison. This requires only moderate space in memory even for large output data. This digest can be thought of as a functional fingerprint of the chunk regarding a projection to one of its output variables. To construct the MD5 digest, we transform each output object to a string representation and append it to the MD5 digest. We use the XStream XML serialization library ${ }^{2}$ to transform an arbitrary object into a string with its XML serialization. The MD5 digest of each chunk is used as a key into a hash map holding the chunks. If two chunks have the same MD5 digest, we have identified a pair of functionally similar code chunks. This is done for eliminating the otherwise quadratic effort of comparing all chunks for equal MD5 digests. While hash collisions could lead to false positives, we consider the comparison correct, since collisions are very unlikely in practice. The result of the output comparison step is a set of equivalence classes of chunks with similar functionality.

\section{E. Post Processing}

Subsumption filter: An additional filter discards simions that are entirely covered by a larger simion (in terms of its length and position in the source code). For example, if two methods are identified to be simions, it is usually not worth reporting that parts of them are also simions.

Type-3 clone filter: Additionally, at the end of the pipeline a type-3-clone clone detector is run to determine which of the simions could also be detected by a type-3-clone-detector, $i$. $e$., a clone detector that takes into account insertion, modification, and deletion of a certain amount of statements. The filter calculates the statement-level edit-distance between chunks and is configured to filter all chunks with an edit-distance less or equal to 5 . Both clone filters are implemented with ConQAT's clone detection algorithm [6]. We cannot filter type3 clones earlier, as they are not guaranteed to be functionally

\footnotetext{
${ }^{2}$ http://xstream.codehaus.org
}

equivalent. Thus, it is unclear which of the instances should be filtered as each could be a potential simion of another chunk.

\section{STUdy Design}

\section{A. Research Questions}

RQ 1: How large is the simion detection problem?

As a first step, we are interested to characterize the problem we want to solve. We look at the number of chunks extracted by our approach.

RQ 2: How do technical challenges affect the detection?

To compare the functionality of two chunks, we need to transform them into executable code pieces and generate useful test cases. This provokes a number of technical challenges that affect the detection approach. This includes the generation of input values for the chunks, which need to be meaningful to trigger interesting functionality. Furthermore, the generation of useful test data for project-specific data types and the emulation of certain operations used by the chunks such as file I/O or GUI events is challenging. Finally, even after overcoming these limitations, there can still be problems that prevent the compilation of the extracted chunk. We investigate how many chunks need to be disregarded during detection because of these challenges.

RQ 3: How effective is our approach in detecting simions?

We ask if the approach is able to detect significant amounts of functionally similar code. Moreover, as we investigated the technical challenges in RQ 2, we are interested in the share of the code of real-world systems that we are able to analyze. Finally, we want to know how many simions we can find in realistic systems.

\section{B. Data Collection Procedure}

In Section III we described three different chunking strategies. The general idea is to run a complete simion detection with all strategies on a set of software systems to collect the needed data for answering the research questions. For practical reasons, however, we cannot perform a complete detection using the sliding window chunking strategy, because it creates far more chunks than are feasible to analyze. Therefore, we collect data using that strategy only for RQ1.

For RQ 1 and RQ 2, we employ no filters, since we are only interested in determining the difficulty of the problem and technical challenges regardless of the precision of the results. For RQ 3, where we are interested in the amount of detected simions, we use all filters.

$R Q$ 1: ConQAT writes the total number of extracted chunks into its $\log$ file, which answers RQ 1 . We use separate configurations for the different chunking strategies.

$R Q$ 2: To answer RQ 2, we use two different configurations. The first one is similar to RQ 1, using a specific statistics processor for collecting the required data. Our input generator logs the number of chunks for which no input could be generated. Additionally, our configuration determines and counts the types of the input parameters and aggregates these values. 
Another part of the configuration counts the number of chunks that contain calls to I/O, networking, SQL, or UI code. We cannot execute chunks containing such calls successfully, as the expected files, network peers, or databases are not available, or the required UI initialization was not performed. We identify calls to these groups by the package the corresponding class resides in, e.g., a call to java.io.File.canRead() would be counted as I/O. These packages also contain methods that can be safely called even without the correct environment being set up (such as methods from java.io.StringReader), so we expect to slightly overestimate these numbers. On the other hand, we only count methods that are directly called from the chunk. Methods that are called indirectly (from other methods called from the chunk) are not included in these numbers. However, as we are only interested in the magnitude of this problem, we consider this heuristic sufficient.

The second configuration is a slightly simplified detection pipeline, that uses the approach described in Section III to generate code and tries to compile the chunks. Statistics on the number of chunks that could not be compiled are reported. For both configurations, we disabled the type-1.5 clone filter and the permutation step, as these distort the statistics slightly.

$R Q$ 3: For the last research question we utilize the full simion detection pipeline to count the number of simions detected by our implementation. Our code is instrumented to report the number of chunks lost at the steps of the pipeline.

\section{Analysis Procedure}

$R Q$ 1: For the size of the problem, we report the total number of chunks per chunking strategy to show the order of magnitude. To make the numbers more comparable and to allow an estimate for the simion detection in systems of other sizes, we give the number relative to the lines of code (SLOC) and calculate the mean value.

$R Q$ 2: We show the relative distribution and calculate the mean per strategy for each of the following metrics, which characterize different technical challenges. We give the values for all relative metrics rounded to full percentages. First, we analyze the difficulty of generating inputs by two metrics. One is the number of chunks for whose input parameters we cannot generate values. The other is the number of inputs of projectspecific data types, because it is especially hard to generate meaningful input for them. Second, for the execution there are certain types of methods that are hard to emulate during random testing. We analyze the number and share of calls to I/O, network, SQL, and UI. Third, the chunks need to be compiled to be executed. Hence, we investigate the fraction of chunks that cannot be compiled. For these challenges, we add qualitative, manual analysis of the chunks that cannot be further used in the detection approach to get more insights into the reasons.

$R Q$ 3: We analyze two different types of study objects. The first type is a set of programs of which we know that they have to exhibit similar functionality, because they were produced according to the same specification. These study objects show whether the detection approach works in
TABLE I

Size of Open SOURCE STUdy OBJects

\begin{tabular}{|l|r|}
\hline Project & SLOC \\
\hline \hline Commons Lang & 17,504 \\
\hline Freemind & 51,762 \\
\hline Jabref & 74,586 \\
\hline Jetty & 29,800 \\
\hline JHotDraw & 78,902 \\
\hline \hline Overall & $\mathbf{2 5 2 , 5 5 4}$ \\
\hline
\end{tabular}

principle. We expect to get at least as many simions reported as there are implementations of the specification. The second type of study objects are real-world, large systems of which we do not know beforehand of any simions. We show the change in chunks during the execution of the detection pipeline in absolute and relative terms. We round the relative values to percentages with two positions after the decimal point to be able to differentiate small results.

\section{Study Objects}

We chose two different types of study objects: (1) a large number of Java programs that are functionally similar and (2) a set of real-world Java systems. The study objects of type 1 are small programs, so that they are easy to analyze and they are built according to the same specification so that we can be sure that they exhibit largely the same functionality. We selected a set of programs, we used in an earlier study [4]. We will refer to it as "Info1". They are implementations of a specification about an e-mail address validator by computer science undergraduate students. We only include programs passing a defined test suite to ensure a certain similarity in functionality. This results in 109 programs with a size ranging from 8 to 55 statements.

The second type of study objects represents real Java systems to show realistic measurements for chunks and simions. As selection criteria, we chose systems that cover a broad range of application domains, sizes, and functionalities. Furthermore, we chose systems we are already familiar with to support the interpretation of the results. The selection resulted in the five open source Java systems that represent libraries, GUI applications, and servers, shown in Table I together with their size in SLOC (source lines of code, i.e., the number of non-blank and non-comment lines).

\section{Results}

\section{A. RQ 1: Problem Size}

Table II shows the absolute and relative numbers of chunks extracted for each study object and the different chunking strategies. The sliding window strategy extracts the highest number of chunks with up to 2.68 chunks per SLOC and 1.44 chunks/SLOC on average. The intent-based strategy creates less chunks with at most 0.40 chunks/SLOC and 0.25 chunks/SLOC on average. The smallest number of chunks is extracted using the method-based strategy. It creates at most 0.09 chunks/SLOC and 0.05 chunks/SLOC on average. 

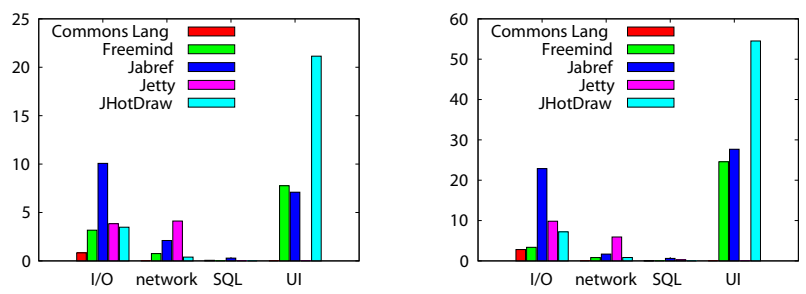

Fig. 2. Fraction of chunks calling complex methods $(\%)$ for method chunking (left) and intent chunking (right)

Overall, the number of chunks is large, especially for the sliding window strategy. The detection approach needs to be able to cope with several thousand chunks.

\section{B. $R Q$ 2: Technical Challenges}

The first pair of columns in Table III shows the fraction of chunks for which the approach cannot construct input values. The two main cases where no input can be generated are chunk parameters that refer to (1) an interface or abstract class and (2) a collection with an unknown component typ $\mathrm{s}^{3}$ In the first case it is unclear which implementation should be chosen to obtain an object that implements the interface. In the second case we do not know what type of objects to put in the collection. For all systems the fraction for which no input can be generated is higher for the intent-based chunking strategy compared to the method-based strategy. In case of Freemind and the intent-based chunking strategy for as much as $94 \%$ of the chunks no input could be generated. An investigation revealed that Freemind uses untyped Collections. Except for Commons Lang, where primitive types are dominant, input generation failed for more than $30 \%$ of the chunks with both chunking strategies.

We determined how many chunks have parameters (either input or output) of project-specific data types. The results are shown in the second pair of columns in Table III A considerable fraction of the chunks (60-87\%) refer to projectspecific data types. With our approach, these would not qualify as candidates for cross-project simions, since the other project would not have the same data types.

To estimate the use of methods that require a specific environment, we determined for each chunk whether it contains direct calls to methods from one of the groups I/O, networking, SQL, or UI. The relative numbers of chunks containing calls to a category are shown in Figure 2 for each of the categories. It is not surprising, that the numbers depend on the application. The library Commons Lang has only a couple of calls to I/O code, the HTTP server Jetty has the highest number of calls to networking code, and the drawing tool JHotDraw dominates the UI group. The most common groups are UI (if the application has a user interface at all), followed by I/O.

In the execution step, a chunk containing methods from at least one of these groups is likely to fail as the expected

\footnotetext{
${ }^{3}$ This second issue especially applies to code that is not targeted at version 1.5 or above of Java which supports Generics and thereby allows to specify the component type within the declaration of a collection type.
}

environment is not provided. The last pair of columns in Table III lists how many chunks are affected by such methods for both of the chunking strategies. Overall, as many as $24 \%$ of the methods can be affected (JHotDraw), or for the intentbased chunking strategy more than $60 \%$ of the chunks, thus having a significant impact on the number of chunks we can process by our dynamic approach. Interestingly, the relative numbers for the intent-based strategy are higher than for the method-based strategy in all cases. This suggests, that the methods containing I/O or UI code are typically longer than the remaining methods and thus produce more chunks.

Finally, we checked how many of the chunks we were able to make compilable by providing a suitable context. The relative number of chunks we could make compilable of those for which at least one input could be generated is shown in Table IV These numbers do not indicate a principal limitation, as each of the chunks we extracted is a valid subtree of the AST and thus can be executed in a suitable context. They rather document limitations in our tool. An inspection of the problematic chunks revealed weaknesses in chunks dealing with generic data types, anonymous inner classes, method local classes, and combinations thereof. Still, we are able to automatically generate a context for at least $82 \%$ in all cases and up to $99 \%$ for Freemind. Clearly, these numbers could be improved by using more advanced algorithms for generating the context for a chunk. The other results from RQ 2 and those of RQ 3 presented next suggest, however, that the chunks we lost as we cannot make them compile is not the main bottleneck of the detection pipeline.

\section{C. $R Q$ 3: Simions}

Tables V]and VI summarize the analysis results for all study objects discussed previously plus the additional "Info1" system (gray column). For each study object the table depicts all pipeline steps along with the number of resulting chunks after the step's execution (column "Abs."). Additionally, column "Rel." shows the relative number of chunks with respect to the original chunk number created by the chunk extraction step. The absolute delta between the rows shows how many chunks are "lost' ${ }^{4}$ in each pipeline step. The tables' last row "Type-3Clone" reports the total number of simions found after all steps have been processed. We do not report exact processing times, as the load of the machines we were using for the analysis varied. Each individual run for one system took between 1 and 30 hours (depending on number of chunks).

For the Infol data set, we found 105 resp. 418 simions. The higher number for the intent-based strategy is expected, as certain sub-steps of the implementation can be also seen as individual simions that are not removed by the subsumption filter if they occur more often than the surrounding simion. As the data set consists of 109 implementations of the same functionality, we would not expect to find substantially more simions with any other approach. Hence, the recall for purely

\footnotetext{
${ }^{4}$ In the permutation step, additional chunks are created.
} 
TABLE II

Total Number of Chunks For Different Extraction Strategies

\begin{tabular}{|l|r|r|r|r|r|r|}
\hline Object & \multicolumn{2}{|c|}{ Sl. Win. } & \multicolumn{2}{|c|}{ Intent } & \multicolumn{2}{|c|}{ Method } \\
\hline & Total & per SLOC & Total & per SLOC & Total & per SLOC \\
\hline \hline Commons Lang & 7,940 & 0.45 & 1,843 & 0.11 & 1,538 & 0.09 \\
\hline Freemind & 80,816 & 1.56 & 20,632 & 0.40 & 1,984 & 0.04 \\
\hline Jabref & 133,556 & 1.79 & 21,388 & 0.27 & 2,085 & 0.03 \\
\hline Jetty & 22,006 & 0.74 & 7,713 & 0.26 & 1,457 & 0.05 \\
\hline JHotDraw & 211,283 & 2.68 & 16,221 & 0.21 & 2,813 & 0.04 \\
\hline \hline Mean & - & 1.44 & - & 0.25 & - & 0.05 \\
\hline
\end{tabular}

TABLE III

FRACTION OF CHUNKS WITH TECHNICAL CHALLENGES

\begin{tabular}{|l|r|r|r|r|r|r|}
\hline \multirow{2}{*}{ Project } & \multicolumn{2}{|c|}{ No Input } & \multicolumn{2}{|c|}{ Proj. Specific } & \multicolumn{2}{|c|}{ Specific Env. } \\
& Meth. & Int. & Meth. & Int. & Meth. & Int. \\
\hline \hline Commons Lang & $20 \%$ & $37 \%$ & $60 \%$ & $60 \%$ & $1 \%$ & $5 \%$ \\
\hline Freemind & $65 \%$ & $94 \%$ & $79 \%$ & $75 \%$ & $11 \%$ & $27 \%$ \\
\hline Jabref & $32 \%$ & $41 \%$ & $61 \%$ & $81 \%$ & $17 \%$ & $44 \%$ \\
\hline Jetty & $36 \%$ & $66 \%$ & $72 \%$ & $87 \%$ & $7 \%$ & $15 \%$ \\
\hline JHotDraw & $43 \%$ & $55 \%$ & $76 \%$ & $86 \%$ & $24 \%$ & $60 \%$ \\
\hline
\end{tabular}

TABLE IV

COMPILEABLE ChUnKS

\begin{tabular}{|l|r|r|}
\hline Project & Method & Intent \\
\hline \hline Commons Lang & $96 \%$ & $97 \%$ \\
\hline Freemind & $92 \%$ & $99 \%$ \\
\hline JabRef & $90 \%$ & $82 \%$ \\
\hline Jetty & $83 \%$ & $88 \%$ \\
\hline JHotDraw & $93 \%$ & $84 \%$ \\
\hline
\end{tabular}

algorithmic code (no I/O, no custom data structures, etc.) is good.

Overall (not including the Infol set), the analysis discovered 153 simions with the method-based chunking strategy and 294 simions with the intent-based strategy. Compared to the size of the analyzed systems, this number seems small. Furthermore, a manual inspection of a sample of the reported simions revealed that the results still contain false positives, i.e., chunks that a developer would not consider functionally similar. These were in most cases functions that produce the same output for a small number of input values. However, these were mostly "trivial" corner cases regarding the input data (e.g., null or the empty String) and the manual inspection of these functions revealed that they differ significantly in the behavior for "interesting" input data values, thus not qualifying as simions according to our definition. Examples for valid simions included functions that used different data types (such as primitive types and their corresponding wrapper types) but performed an equal function. Other valid simions were functions that simply delegated to another one (in some cases with additional error handling in the delegating function), thus trivially exhibiting equal functionality.

RQ 2 quantified the numbers of chunks that are discarded due to different technical reasons, such as missing input. A chunk can be affected, however, by more than one of these issues. To understand how many chunks are affected by none of these problems, we have to look at the entire processing pipeline. On average, about $28 \%$ of chunks for both strategies survive the execution step and all preceding steps. This is still a significant part of the systems in which we can in principle find simions using our approach. Yet, this also means that more than two thirds of the chunks are lost before the actual comparison can be performed and is thus not receptive for our dynamic detection.

Another observation is that while the intent-based chunking strategy results in the higher number of simions found, the relative number of simions found in comparison to the number of input chunks is four times higher for the method-based strategy. One explanation is that the method boundary chosen by the developer is much more likely to encapsulate a reusable fragment. Fragments from within a method are more likely to not be sensible for another developer, thus the probability of finding a duplicate of it is lower. This means that the intentbased strategy is preferred in terms of results (more simions), but compared to the required computation time (which scales linear in the number of chunks) the method-based approach is more effective.

The tables also hint at the amount of cloning. In the methodbased case on average $27 \%$ of the chunks are discarded early on as type- 1.5 clones (for the intent-based strategy this number is lower with about $4 \%$ ). From the simions found, $41 \%$ (or $22 \%$ for intent-based) could be found by a type- 3 clone detector. The absolute numbers provide an even clearer picture. There are 17 times as many chunks removed by the type-1.5 clone filter as there are simion instances reported (9 times for intent-based), so the cloning problem seems to be worse than the problem of independent reimplementation (simion). Additionally, clones (even of type-3) are much easier to detect than simions (both in terms of the involved algorithms and the required processing time) and the detection of clones is also possible for code where we can not find suitable input or a compilation context. From a quality improvement standpoint, this indicates that detecting simions should only be performed, after all clones (the "low hanging fruits") have been filtered.

\section{Threats TO VALIDITY}

One possible threat to the validity of our results are errors in our implementation of the detection pipeline. To mitigate this, we integrated excessive logging in our tool and inspected samples of the reported or excluded chunk at every pipeline step during development. Additionally, we included the study 
TABLE V

ANALYSIS RESULTS FOR THE METHOD-BASED CHUNKING STRATEGY

\begin{tabular}{|c|c|c|c|c|c|c|c|c|c|c|c|c|}
\hline & \multicolumn{2}{|c|}{ Comm. Lang } & \multicolumn{2}{|c|}{ Freemind } & \multicolumn{2}{|c|}{ JabRef } & \multicolumn{2}{|c|}{ Jetty } & \multicolumn{2}{|c|}{ JHotDraw } & \multicolumn{2}{|c|}{ Info1 } \\
\hline & Abs. & Rel. [\%] & Abs. & Rel. [\%] & Abs. & Rel. [\%] & Abs. & Rel. [\%] & Abs. & Rel. [\%] & Abs. & Rel. [\%] \\
\hline Chunk Extr. & 1,538 & 100.00 & 1,984 & 100.00 & 2,085 & 100.00 & 1,457 & 100.00 & 2,813 & 100.00 & 240 & 100.00 \\
\hline Type-1.5-Clone & 1,100 & 71.52 & 1,541 & 77.67 & 1,647 & 78.99 & 1,034 & 70.97 & 1,936 & 68.82 & 230 & 95.83 \\
\hline Permutation & 1,601 & 104.10 & 1,916 & 96.57 & 2,245 & 107.67 & 1,255 & 86.14 & 3,472 & 123.43 & 231 & 96.25 \\
\hline Input Gen. & 1,265 & 82.25 & 643 & 32.41 & 1,542 & 73.96 & 742 & 50.93 & 1,879 & 66.80 & 134 & 55.83 \\
\hline Compilation & 1,215 & 79.00 & 530 & 26.71 & 1,324 & 63.50 & 568 & 38.98 & 1,586 & 56.38 & 133 & 55.42 \\
\hline Execution & 1,066 & 69.31 & 189 & 9.53 & 621 & 29.78 & 313 & 21.48 & 660 & 23.46 & 133 & 55.42 \\
\hline Identity & 1,066 & 69.31 & 189 & 9.53 & 621 & 29.78 & 313 & 21.48 & 660 & 23.46 & 133 & 55.42 \\
\hline Equality & 947 & 61.57 & 165 & 8.32 & 522 & 25.04 & 178 & 12.22 & 579 & 20.58 & 133 & 55.42 \\
\hline Comparison & 108 & 7.02 & 15 & 0.76 & 94 & 4.51 & 19 & 1.30 & 34 & 1.21 & 105 & 43.75 \\
\hline Subsumption & 108 & 7.02 & 13 & 0.66 & 90 & 4.32 & 19 & 1.30 & 30 & 1.07 & 105 & 43.75 \\
\hline Type-3-Clone & 54 & 3.51 & 11 & 0.55 & 55 & 2.64 & 15 & 1.03 & 18 & 0.64 & 105 & 43.75 \\
\hline
\end{tabular}

TABLE VI

ANALYSIS RESULTS FOR THE INTENT-BASED CHUNKING STRATEGY

\begin{tabular}{|c|c|c|c|c|c|c|c|c|c|c|c|c|}
\hline & \multicolumn{2}{|c|}{ Comm. Lang } & \multicolumn{2}{|c|}{ Freemind } & \multicolumn{2}{|c|}{ JabRef } & \multicolumn{2}{|c|}{ Jetty } & \multicolumn{2}{|c|}{ JHotDraw } & \multicolumn{2}{|c|}{ Info1 } \\
\hline & Abs. & Rel. [\%] & Abs. & Rel. [\%] & Abs. & Rel. [\%] & Abs. & Rel. [\%] & Abs. & Rel. [\%] & Abs. & Rel. [\%] \\
\hline Chunk Extr. & 1,843 & 100.00 & 20,632 & 100.00 & 21,388 & 100.00 & 7,713 & 100.00 & 16,221 & 100.00 & 1,969 & 100.00 \\
\hline Type-1.5-Clone & 1,598 & 86.71 & 20,586 & 99.78 & 20,794 & 97.22 & 7,527 & 97.59 & 14,643 & 90.27 & 1,938 & 98.43 \\
\hline Permutation & 4,690 & 254.48 & 26,705 & 129.43 & 61,836 & 289.12 & 17,131 & 222.11 & 51,683 & 318.62 & 3,259 & 165.52 \\
\hline Input Gen. & 2,823 & 153.17 & 2,858 & 13.85 & 33,559 & 156.91 & 5,301 & 68.73 & 26,347 & 162.43 & 1,910 & 97.00 \\
\hline Compilation & 2,772 & 150.41 & 2,432 & 11.79 & 22,837 & 106.77 & 4,227 & 54.80 & 16,527 & 101.89 & 1,883 & 95.63 \\
\hline Execution & 2,399 & 130.17 & 556 & 2.69 & 3,998 & 18.69 & 2,173 & 28.17 & 9,887 & 60.95 & 1,841 & 93.50 \\
\hline Identity & 2,298 & 124.69 & 424 & 2.06 & 3,480 & 16.27 & 1,734 & 22.48 & 9,352 & 57.65 & 1,825 & 92.69 \\
\hline Equality & 2,014 & 109.28 & 388 & 1.88 & 2,818 & 13.18 & 1,392 & 18.05 & 8,694 & 53.60 & 1,730 & 87.86 \\
\hline Comparison & 85 & 4.61 & 4 & 0.02 & 232 & 1.08 & 36 & 0.47 & 131 & 0.81 & 752 & 38.19 \\
\hline Subsumption & 77 & 4.18 & 4 & 0.02 & 196 & 0.92 & 32 & 0.41 & 67 & 0.41 & 420 & 21.33 \\
\hline Type-3-Clone & 59 & 3.20 & 2 & 0.01 & 159 & 0.74 & 28 & 0.36 & $\overline{46}$ & 0.28 & 418 & 21.23 \\
\hline
\end{tabular}

objects from [4], for which the number of simions is known, into our study objects. This ensures that our approach and implementation are capable of finding at least certain simions.

To improve the validity of the results we chose study objects we were familiar with from earlier experiments in a code analysis and quality context. This helped us to interpret the results compared to an entirely unknown system. Still, we attempted to select study objects of different types and sizes, to improve transferability of our results to other Java systems.

An internal threat to validity is that the different filters used can distort the results for individual technical challenges. We mitigated this threat by separate configurations for collecting different data. The configuration for the results of RQ 2 uses less filters so we could get the complete results.

\section{RELATED WORK}

Work related to ours can be found in the area of clone detection (for an overview, see [1], [2]), where syntactically similar code is searched. As the techniques used there only work on a textual semi-structured representation, however, they cannot be used to find code fragments that are semantically similar but not syntactically similar as shown in [4]. Another related area is equivalence checking (e.g., [7]-[9]), which is a wellstudied but undecidable problem. While these papers provide a theoretical foundation, they do not provide techniques for finding equivalent code in large real-world systems. We also use techniques known from random testing, such as [10], but our setting is different as we require the same input for many test cases (chunks).

\section{A. Comparison to Jiang\&Su}

To the best of our knowledge, the work of Jiang\&Su [5] is the only published attempt of automatically mining semantically equivalent code from a large code base. Thus, we compare our approach and results in detail to their paper in this section. The most obvious difference is that our tool chain works on Java code, while their tool, called EqMiner, deals with the $\mathrm{C}$ language. For a more systematic comparison, we structure the comparison according the different phases of the detection pipeline.

Chunk Extraction Phase: This phase is called code chopper in EqMiner. Their approach corresponds to the sliding window chunking strategy with a minimal window size of 10 statements. They report that for long functions the quadratic number of chunks created this way is too large, which matches our observation. To mitigate this, EqMiner "randomly selects up to 100 code fragments from all code fragments in each function". We expect, that this random selection can cause relevant chunks to be missed. Thus, we employ a strategy based on logical separation found in the syntax, which also helps to reduce the number of chunks, but hopefully better captures the programmer's intent compared to random chopping.

Input Generation Phase: The type system of $\mathrm{C}$ essentially consists of primitive types, pointers, and record types (structs). 
Consequently, the input generator used in EqMiner supports generation of all of these types, including dynamic allocation of structs to provide a pointer to a struct, but the generation of arrays is not supported. In Java, there are also primitive types and arrays, but instead of structured data (structs) and pointers, Java has classes and object references. While similar in the intent, this complicates input generation. Furthermore, the presence of abstract types and interfaces leads to situations where a suitable concrete implementation can not be easily generated as input. As long as non-abstract classes are used, we follow the approach from [10] by picking a random constructor and recursively generating inputs for its parameters.

Chunk Execution Phase: This phase in our tool corresponds to the code transformation and code execution steps in EqMiner. The obvious difference is that to make a fragment of Java code compile requires slightly different surrounding code than with $\mathrm{C}$ code. Especially the access to local attributes and methods within the same class requires additional considerations. The main difference, however, is how we treat function calls within the extracted chunk. In EqMiner, Jiang\&Su "view each callee as a random value generator and ignore its sideeffects besides assignments through its return values (i.e., the random values)". We found this approach too limiting for Java code, as we might miss interdependencies between method calls (for example code might rely on getting the same value from a getter method that was earlier passed to the corresponding setter method). Instead we just execute the original method, which means that the entire context of the chunk must be reconstructed, including the surrounding class with its attributes and methods. Actually executing the methods also requires protection of the execution environment of unwanted side-effects. For example, feeding code that deletes files with random data could cause problems during analysis. Using Java's security manager, however, we could ensure the program to not cause these kinds of problems.

Result Comparison Phase: EqMiner treats code chunks as equivalent, if they produce the same output for ten different random inputs. Jiang\&Su also report, that a common pattern found in the largest clusters of equivalent code is that the (single) output of the chunk is exactly the input value. So, the chunk is essentially equivalent to the identity (or a projection of inputs). Our experiments also showed this pattern and manual inspection of these chunks revealed that these fragments typically influence the system by other means (for example by calling functions with side-effects) and would not be considered equivalent by a developer. We filtered out all chunks following this pattern prior to comparison. Our tool discards chunks that return the same result for all random inputs. As the results for RQ 3 indicate, both cases are frequent.

Study Objects: Jiang\&Su evaluated their tool on a sorting benchmark and the Linux kernel. Both systems do not contain code that performs I/O operations (actually the kernel offers system calls for performing I/O) or deal with UIs. Additionally, a huge part of the kernel deals with process scheduling, device drivers, or memory management, which are all not known to require complex string processing. Contrary, as shown by RQ 2, our study objects spend lots of code on $\mathrm{I} / \mathrm{O}$ and UI tasks, and string processing is essential for parts of them. As I/O and UI are tricky for input generation, and string algorithms are often hard to differentiate with only 10 inputs, the number of valid chunks and false positives is affected by our choice of systems (which are typical for Java programs).

\section{DISCUSSION}

The low number of simions raises the question whether there are no simions in those systems or rather our detection approach is flawed. Ideally, we would answer this question by calculating the recall, i.e., the fraction of all known simions we are able to detect. For realistic systems, such as our study objects, the number of existing simions is not known (and practically infeasible to determine manually by inspection for even a small part of them). For artificial benchmarks, such as the Infol set, we have a good estimate of the number of simions. Yet, while our recall is good for this study object, the comparison with the numbers from Tables $[\mathrm{V}]$ and $[\mathrm{VI}$ shows that they are not representative for realistic systems.

Intuition and experience tells us, that developers tend to reinvent the wheel and so we would expect many simions. One explanation for the low rates could be that we only analyzed simions within a single system. Maybe developers know their own code base well enough to reuse code (either by referencing or cloning it) instead of reimplementing it as a simion. First experiments with detection of simions between two independent projects, however, did not reveal substantially higher simion rates. One explanation is given by Table III which reports high rates of chunks with project specific data types. As we only find simions with the same I/O signature, these chunks can not be cross-project simions.

In our opinion another reason for the low detection rates is that the notion of $\mathrm{I} / \mathrm{O}$ equivalence is inappropriate. Often, code encountered in practice might be intuitively counted as a simion, but does not exhibit identical I/O behavior. Reasons are differences in special cases, the error handling, or the kind of data types used at the interfaces. An extreme example would be databases from different vendors. While they basically provide the same functionality and interface (SQL), migrating from one database to another is typically far from trivial as the behavior is not the same in all details. Thus, we believe that there should be a better definition of a simion than the I/O behavior of code chunks. Finding a suitable definition and exploiting it for simion detection is one of the main open questions for future work in this area.

\section{CONClusions ANd Future Work}

In this paper, we presented an approach for detecting functionally similar code fragments in Java systems, which was inspired by an existing approach for $\mathrm{C}$ systems. We evaluated the approach for 5 open source systems and an artificial system with independent implementations of the same specification. In contrast to existing work targeting $\mathrm{C}$ systems, we experienced low detection results. In our opinion, this is mainly due to the 
limited capability of the random testing approach. In many cases, input generation either fails to generate valid input or the generated input is not able to achieve sufficient code coverage. There is also reason to believe that similarities are missed due to the chunking, e.g., if code fragments perform a similar computation but use different data structures at their interfaces. Further research is required to quantify these issues. To support further investigation by other researchers, we provide the implementation of our approach for download 5

As future work we plan to manually assess the detected simions in more detail and check whether they really represent simions according to our definition. Furthermore, we intend to apply the approach to detect simions between projects. Another interesting idea is to employ advanced test generation methods, e.g., feedback-directed random testing, or white-box techniques, to achieve higher coverage rates. We also want to reconsider the definition of simions to better reflect the intuitive notion of similar functionality. A different definition might inspire new ways of detection with better results.

\section{Acknowledgments}

We thank the Google Research Awards Program for awarding our proposal "Detecting Behavioral Redundancy and Clones in Software Source Code".

\section{REFERENCES}

[1] R. Koschke, "Survey of research on software clones," in Duplication, Redundancy, and Similarity in Software, 2007.

[2] C. K. Roy and J. R. Cordy, "A survey on software clone detection research," Queen's University, Kingston, Canada, Tech. Rep. 2007-541, 2007.

[3] E. Juergens, F. Deissenboeck, B. Hummel, and S. Wagner, "Do code clones matter?' in ICSE'09, 2009.

[4] E. Juergens, F. Deissenboeck, and B. Hummel, "Code similarities beyond copy \& paste," in CSMR'10, 2010

[5] L. Jiang and Z. Su, "Automatic mining of functionally equivalent code fragments via random testing," in ISSTA'09, 2009.

[6] E. Juergens, F. Deissenboeck, and B. Hummel, "CloneDetective - A workbench for clone detection research (Tool Demo)," in ICSE '09, 2009.

[7] G. Cousineau and P. Enjalbert, "Program equivalence and provability," in Mathematical Foundations of Computer Science 1979, 1979.

[8] J.-C. Raoult and J. Vuillemin, "Operational and semantic equivalence between recursive programs," J. ACM, vol. 27, no. 4, pp. 772-796, 1980

[9] M. Bertran, F.-X. Babot, and A. Climent, "An input/output semantics for distributed program equivalence reasoning," Electr. Notes Theor. Comput. Sci., vol. 137, no. 1, pp. 25-46, 2005.

[10] C. Pacheco, S. K. Lahiri, M. D. Ernst, and T. Ball, "Feedback-directed random test generation," in ICSE'07, 2007.

(C) 2012 IEEE. Personal use of this material is permitted. Permission from IEEE must be obtained for all other users, including reprinting/republishing this material for advertising or promotional purposes, creating new collective works for resale or redistribution to servers or lists, or reuse of any copyrighted components of this work in other works.

5 http://www4.in.tum.de/ ccsm/simions 